\title{
Correction to: Measuring nanoparticles in the size range to $2000 \mathrm{~nm}$
}

\author{
Philip J. Wyatt $\mathbb{D}$
}

Published online: 4 February 2019

(C) Springer Nature B.V. 2019

\section{Correction to: J Nanopart Res https://doi.org/10.1007/s11051-018- 4397-x}

The original version of this article unfortunately contained mistakes. The corrections are given in the following list:

(1) In "Limitations of RG" section, these two paragraphs should be deleted:

Consider now an example where both Eqs. (1) and (2) would appear to exclude any possibility of RG providing a measure of particle size since the refractive index is far beyond any reasonable value: a gold particle of radius $250 \mathrm{~nm}$ and refractive index $0.14246+3.6821 i$. Figure 1 contrasts the exact Lorenz-Mie theory at the wavelength of $658 \mathrm{~nm}$ with the RG approximation of Eq. (7) for the case of such spheres in water $n_{0}=1.33$. For gold at that wavelength, the value of $|m-1|=$ 4.99. The data at the 15 angles indicated by the superimposed cross hatches correspond to the set

The online version of the original article can be found at https://doi.org/10.1007/s11051-018-4397-x

P. J. Wyatt $(\bowtie)$

Wyatt Technology Corporation, 6330 Hollister Avenue, Goleta, CA 93117, USA

e-mail: pwyatt@wyatt.com that would be measured with a commercial MALS instrument (DAWN HELEOS II n.d.) as listed explicitly in Table 1, below.

Returning to the studies by Kerker et al. (1963) testing the applicability of the RG approximation to provide an accurate measure of the scattering by homogeneous spheres, we note that the authors inferred also a reasonable basis for estimating the applicability of the approximation to other particle shapes and forms. As we shall see later in this paper, the analytical extension of the RG approximation to very small scattering angles may permit a simplified means to extract a reasonable approximation of the size of such gold particles. If that is successful, we would expect that a similar approximation theory might well be used to determine structural properties of more complicated particles (with more common refractive indices!) for which an exact theory (such as Lorenz-Mie) does not exist. We shall return to these gold particle exemplars in the final section and confirm that the analytical procedure concept to be presented is both applicable and appropriate.

(2) In "Preparing and measuring selected samples" section, page 4, first paragraph, the first sentence should be rewritten as: Before MALS measurements are 
made at the angles (DAWN HELEOS II) listed in Table 1 to extract particle sizes and related features, appropriate monodisperse samples must be prepared.

Page 5, paragraph 3, lines 19-23, sentence should be rewritten as: Although they are extremely thin rods of diameter about $1 \mathrm{~nm}$, even smaller than cellulose tubes, application of Eq. (6) yields a very poor fit to the measured data, as we shall see presently in Fig. 3.

Page 6, paragraph 5, last two sentences should be rewritten as: An aliquot was then injected for fractionation, producing the elution $\left(90^{\circ}\right.$ scattering versus time/slice number) of Fig. 2. The vertical bar shows the location of slice 979 whose MALS scattering data points are shown in Fig. 3.

Page 6, paragraph 6, the first sentence should be rewritten as: Figure 3 contrasts the ( $n=15$ detectors) data collected at slice 979 with their best fit to the RG rod model of Eq. (6) yielding a length of $189.4 \pm$ $1.6 \mathrm{~nm}$.

(3) In "Analytical extension: the form factor at very small angles" section, page 10, paragraph 3, the first sentence should be rewritten as: In Fig. 3, for example, the scattered intensities, $I\left(\theta_{\mathrm{i}}\right)(i=1,15)$, are shown as collected at $n=15$ distinct values of $\sin ^{2}\left(\theta_{\mathrm{i}} / 2\right)$ from the ensemble of identical singlewall carbon nanotubes. The best fit to the thin rod structure per Eq. (6) is shown.

Page 11, last paragraph, the last sentence should be rewritten as: From the actual data collected (such as the data points shown in Fig. 3), we create function $\Pi m(\theta)$ that best represents the collected data and, from which, we hope to derive its mean square radius $\left\langle r_{g}^{2}\right\rangle$.

(4) In "Examples of the analytical representation by $\Pi m(\theta)$ " section, page 11, paragraph 2, the third to the last sentence should be rewritten as: The best fit corresponds to the smallest ratio, 0.025 , i.e., $m=5$, yielding an $r_{g}$ of 201.5 and radius of $255 \pm 6.6 \mathrm{~nm}$.

Page 12, paragraph 4, the first sentence should be rewritten as: For the next example, we return to Fig. 3 (the collected SWCNT data for slice 757 fit to the rod model of the RG approximation) and the determination of the function $\Pi m(\theta)$ that will fit these data best.

Page 13, paragraph 6 , the fourth sentence should be rewritten as: This fit does not even appear more accurate than the thin rod model value of $189 \mathrm{~nm}$ from Fig. 3, so higher orders should be examined.
Page 14, paragraph 8, the first and second sentences should be rewritten as: As a final example, consider where both Eqs. (1) and (2) would appear to exclude any possibility of RG providing a measure of particle size since the refractive index is far beyond any reasonable value: a gold particle of radius $250 \mathrm{~nm}$ and refractive index $0.14246+3.6821 i$. Figure 13 contrasts the exact Lorenz-Mie theory at the wavelength of $658 \mathrm{~nm}$ with the RG approximation of Eq. (7) for the case of such spheres in water $n_{0}=1.33$. For gold at that wavelength, the value of $|m-1|=$ 4.99. The data at the 15 angles indicated by the superimposed cross hatches correspond to the set that would be measured with the MALS instrument as listed explicity in Table 1 . The estimated experimental errors of the water solvent per, for example, those measured during the collection of the data shown in Fig. 8, is added to yield Table 5 below.

(5) In "Summary and conclusions" section, page 16, paragraph 4, the first sentence should be rewritten as: Despite some uncertainties of the actual mechanisms by which rod-like particles are separated in A4F devices (Park and Mittal 2015; Nguyen et al. 2013), under a variety of conditions, separations by length actually do occur [Cf. Fig. 3].

Page 16, paragraph 5, the second sentence should be rewritten as: Thus the SWCNT particles separated at each slice, as indicated by the slice profile example of Fig. 2, should be similarly collected and measured.

(6) Figures 2 and 3 were interchanged. The correct Figs. 2 and 3 are now given below:

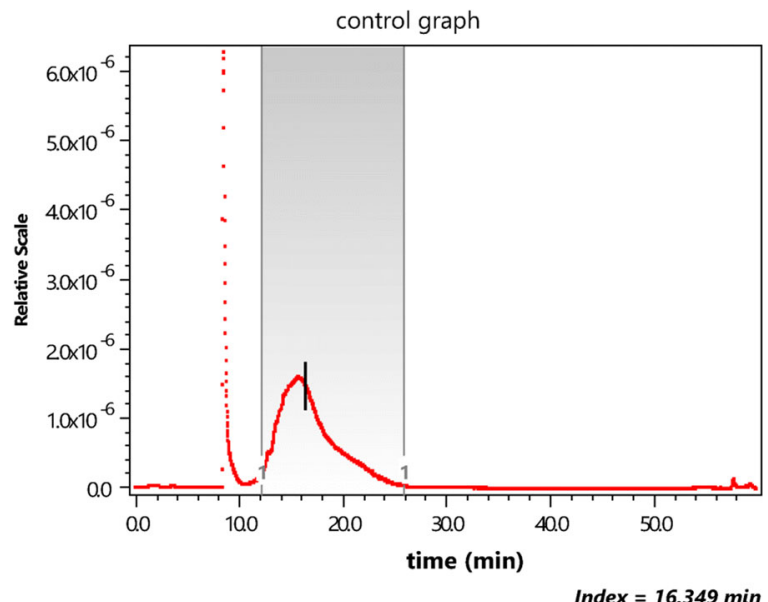

Fig. 2 The scattered intensity at $90^{\circ}$ from a suspension of the NIST short fraction as a function of elution time (seconds) during an A4F fractionation 


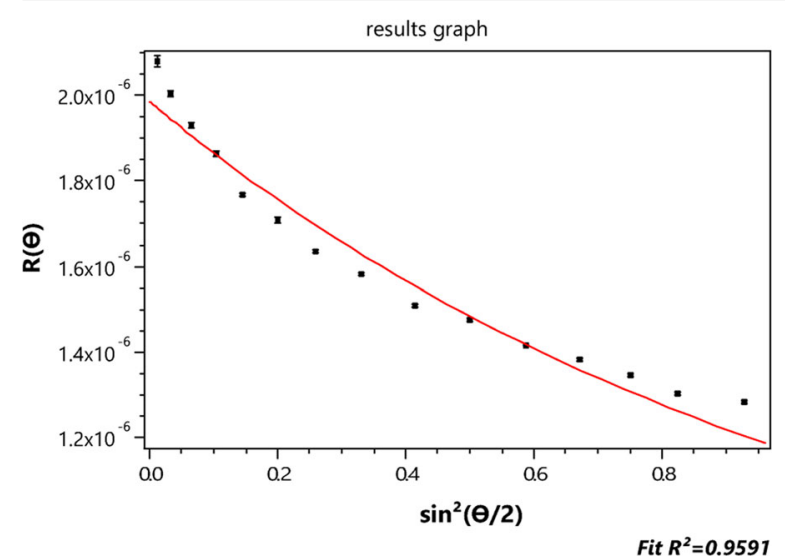

Fig. 3 Best fit of the collected 15 angle scattering data to the RG rod model of Eq. (6); $\mathrm{R}(\theta) \mathrm{vs}$. $\sin 2(\theta / 2)$
(7) In Fig. 11, the caption should be rewritten as: The data of Fig. 3 compared to the fit of function $\Pi_{2}$ yielding an $r_{g}$ of $58.8 \pm 2$

(8) In Fig. 12, the caption should be rewritten as: The data of Fig. 3 compared to the fit of function $\Pi_{3}$ yielding an $r_{g}$ of $70.7 \pm 2.4$

The original article has been corrected.

Publisher's note Springer Nature remains neutral with regard to jurisdictional claims in published maps and institutional affiliations. 